\title{
El voluntariado corporativo y sus efectos sobre la satisfacción laboral y el compromiso en empresas familiares de Andalucía
}

\section{The effect of corporate volunteering programs on job satisfaction and commitment in Andalusian family business enterprises.}

\author{
José Luis Ruizalba Robledo ${ }^{\mathrm{a}^{*}} \cdot$ María Vallespín Arán ${ }^{\mathrm{a}} \cdot J_{\text {osé Luis González Porras }}^{\mathrm{b}}$ \\ ${ }^{a}$ Departamento de Economía y Administración de Empresas. Universidad de Málaga (Málaga) \\ ${ }^{b}$ Universidad de Málaga (Málaga)
}

D A T OS A R TÍ C U L O

Historial:

Recibido 17-12-2013

Aceptado 17-02-2014

Palabras clave:

Responsabilidad Social

Corporativa

Voluntariado Corporativo

Satisfacción laboral

Compromiso

Códigos JEL:

M14, M12, M31

\section{A R T I C LE INFO}

\section{Article history:}

Received 17-12-2013

Accepted 17-02-2014

Keywords:

Corporate Social

Responsibility

Corporate Volunteering

Job Satisfaction

Commitment

JEL codes:

M14, M12, M31

\section{RES U MEN}

Esta investigación analiza el voluntariado corporativo en empresas familiares de Andalucía y su impacto sobre los empleados. Se ha investigado la presencia de programas de voluntariado corporativo junto con otros factores (el interés de la dirección y la conciliación entre vida laboral y familiar) desde la perspectiva de la dimensión de la Responsabilidad Social Corporativa. Tras la revisión teórica, se mide el grado de implantación del voluntariado corporativo en empleados, en clientes y en su intención de desarrollo en el futuro. Queda de manifiesto que las empresas que pongan en marcha acciones de voluntariado corporativo podrían contribuir a incrementar los niveles de satisfacción y de compromiso de los empleados.

A B S T R A C T

This research shows an analysis of the presence of Corporate volunteering programs in family firms of Andalucía (Spain) and its impact on employees. Corporate Volunteerism has been analyzed from social dimension of CSR and how other factors, like management concern and work family balance have impact over job satisfaction and employee's commitment. After theoretical review, Coorporate Volunteerism in Andalusian family enterprises have been measured regarding employees, customers and also the intention of broader implementation in the future. It concludes by stressing the fact that companies that implement corporate volunteerism might help to increase levels of job satisfaction and employee's commitment.

\footnotetext{
* Autor de contacto.

Correoselectrónicos:jruizdealba@uma.es,mvallespin@uma.es,joseluis.gp@hotmail.es
} 


\section{Introducción}

La reciente crisis financiera mundial ha aumentado los niveles de desempleo y pobreza en muchos países. Junto a las duras medidas de recorte que están tomando muchas empresas, paralelamente se observa una mayor sensibilización hacia problemas sociales. De un enfoque centrado en producto, las empresas se fueron orientando hacia el consumidor. Incluso desde hace pocos años se observa una mayor orientación hacia las personas: la rentabilidad se entiende mejor si está equilibrada con la Responsabilidad Social Corporativa (Kotler et al., 2010). Según estos autores, hay significativos ejemplos a nivel mundial de empresas que tienen claros objetivos de contribuir a implementar modelos de negocio centrados en el ser humano y que buscan resolver problemas relacionados con el bienestar, la pobreza y la sostenibilidad medioambiental.

En estos momentos en que están siendo cuestionados los fundamentos de muchos modelos basados en la maximización del beneficio y la deshumanización de las empresas, el debate es intenso a nivel político, social y también académico. En este sentido, la implantación de políticas de Responsabilidad Social Corporativa (en adelante RSC) ha supuesto un gran avance en muchas empresas. Porter y Kramer (2011) proponen la conveniencia de implantar sistemas de Creación de Valor Compartido mediante políticas y prácticas operativas que fomenten la competitividad de las empresas y al mismo tiempo supongan una mejora de las condiciones económicas y sociales de las comunidades en las que operan las empresas. Señalan varias formas de Creación de Valor Compartido e indudablemente el voluntariado corporativo sería una de ellas en la medida en que contribuyen a la creación de valor social.

Pensamos que esta investigación está justificada por la necesidad de conocer el impacto que las acciones de voluntariado corporativo ejercen sobre los empleados en términos de compromiso y de satisfacción laboral ya que se trata de una dimensión que aún no ha sido suficientemente estudiada en el contexto de empresas familiares (Benavides, Guzmán y Quintana, 2011). Además, nuestra aportación proviene del hecho de incorporar el voluntariado corporativo en el marco de las prácticas de Marketing Interno. De este modo, no solo se analiza el impacto del voluntariado corporativo sobre los empleados, sino que se compara con el impacto que tienen otras acciones de Marketing Interno como el Interés de la Dirección y la Conciliación de la vida laboral y familiar.
El documento se ha estructurado como sigue. En primer lugar, se analiza el concepto de voluntariado corporativo en el contexto de la RSC. En segundo lugar, se muestra la situación del análisis del voluntariado corporativo en las empresas españolas. En tercer lugar, se exponen los resultados de una investigación empírica realizada en empresas familiares de Andalucía en la que se analiza la incidencia de algunas variables relativas a la dimensión social de la RSC, como el voluntariado corporativo, sobre la satisfacción y el compromiso organizacional de los empleados. Además, se finaliza presentando algunas conclusiones $y$ propuestas para el ámbito académico y empresarial.

\section{El voluntariado corporativo en el contexto de la RSC}

Desde hace años, la RSC tiene implantación en todo tipo de empresas. Recientemente algunas están además comenzando a diseñar e implementar planes y accionesde voluntariado corporativo que podrían enmarcarse dentro de sus políticas de RSC procurando obtener ventajas en sus propias empresas, en los empleados, en sus clientes y en la sociedad.

\subsection{Concepto de RSC}

Aunque no se cuenta con una definición comúnmente aceptada de RSC, su idea central se construye con la premisa de que las empresas tienen responsabilidad hacia sus stakeholders y también son responsables de su impacto social (Moon, 2011).

El interés académico por la RSC ha evolucionado en paralelo con la intensificación de las iniciativas empresariales en este campo, teniendo la desatención tradicional hacia estos temas su punto de inflexión en los años noventa (Joyner y Payne, 2002). Se deduce que problemas como el deterioro medioambiental imputable a las actividades económicas, las prácticas laborales discriminatorias, la violación de los derechos humanos y el subdesarrollo son cuestiones de hondo calado que preocupan a las empresas socialmente responsables.

De este modo, las empresas que tienen un comportamiento socialmente responsable diseñan sus estrategias y establecen procedimientos internos de gestión teniendo en cuenta no sólo la dimensión económica de sus acciones sino también la social y la medioambiental (Nieto y Fernández, 2004). Por lo tanto, se puede afirmar que el concepto de Responsabilidad Social está relacionado con parte de 
la actividad voluntaria de las empresas relativa a la mejora de la sociedad a la que pertenecen y a la preocupación por el medio ambiente en el que se encuentran (Servero y Villalonga, 2005).

La RSC considera el impacto de las acciones de la empresa en la sociedad y supone que la corporación tiene responsabilidades que van más allá de las obligaciones económicas y legales (Cabrera et al., 2011). Por lo tanto, la adopción del concepto de RSC supone el reconocimiento por parte de las empresas de su responsabilidad hacia la sociedad de ir más allá de la mera maximización de los beneficios (Reynolds, 2013). También es importante señalar que la respuesta a esa responsabilidad se asume o no libremente por las empresas, a las que nadie les obliga en sentido estricto a implantar acciones de RSC, aunque se presente como una potente herramienta de gestión (Carroll, 1999).

Para la AECA (2004), la RSC es el compromiso voluntario de las empresas con el desarrollo de la sociedad y la preservación del medio ambiente, desde su compromiso social y un comportamiento responsable hacia las personas y grupos sociales con quienes interactúa. Esta Asociación destaca también dos ámbitos diferenciados que abarca la RSC, por un lado el compromiso, la asunción de responsabilidades, y por otro, el comportamiento, la acción, la forma de llevar a cabo dichos planteamientos. Dos ámbitos, por tanto, claramente diferenciados: responsabilidad en el plano del compromiso y mentalización (plano estratégico) y comportamiento en el plano de la acción (sistemas de dirección y gestión).

En este sentido, habitualmente suele definirse a la RSC como una variable que incorpora tres bloques teóricos de análisis que determinan la orientación del empresario hacia la RSC: la dimensión económica (desarrollo armónico), la dimensión social (reducción de las desigualdades) y la dimensión medioambiental (medioambiente sano y equilibrado).

Por tanto, mientras que la dimensión económica recoge las prácticas relacionadas con una cadena de suministro sostenible y la dimensión medioambiental comprende iniciativas para mitigar los impactos medioambientales, la dimensión social de la RSC recoge prácticas, tales como la contratación de personas en riesgo de exclusión social, la mejora de la calidad de vida de los empleados, el estar comprometidos con la creación de empleo, el fomento del desarrollo profesional de los empleados, el mantenimiento y la mejora de la calidad de vida en la comunidad local o el apoyo a causas sociales (Gallardo et al., 2013).

\subsection{El voluntariado corporativo como parte de la $R S C$}

El voluntariado corporativo emerge como una herramienta importante a través de la cual las empresas manifiestan su RSC (Gallardo et al., 2010; Vázquez et al., 2010; Basil et al., 2011). Como fenómeno definible, el voluntariado corporativo surge a final de la década de los setenta en los Estados Unidos y en las siguientes tres décadas se extiende lenta pero inexorablemente por todo el mundo (Sanz et al., 2010). Este desarrollo de las políticas de voluntariado corporativo podría encajar con la consideración (Kotler et al., 2010) de que la confianza se está desarrollando más en las relaciones horizontales que en las verticales, de manera que los consumidores creen más en unos y otros que en las propias empresas.

Peterson (2004) recoge la definición de Wild (1993) sobre voluntariado corporativo que lo considera como cualquier tipo de apoyo formal por parte de la empresa para facilitar a los empleados y a sus familias el poder dedicar su tiempo y habilidades en algún tipo de servicio a la comunidad. Por tanto, está enmarcado dentro de un conjunto de comportamientos o acciones de soporte a sus empleados para que puedan implicarse en acciones de voluntariado. Como vemos, esta definición se centra en el voluntariado corporativo dirigido a los empleados, pero también es cada vez más frecuente que las empresas implanten estos planes orientados a implicar a sus clientes. Dichas acciones de voluntariado que implican a los clientes podrían enmarcarse, por tanto, dentro del marketing relacional.

Por ejemplo en España, la Federación de Bancos de Alimentos, ha conseguido que muchas empresas consigan involucrar a sus empleados y clientes en sus campañas de recogida de alimentos. Por otro lado, grandes corporaciones como IKEA, BBVA, McDonald's y Starbucks han implantado estrategias de voluntariado corporativo, en el marco de sus políticas de RSC, llegando a insertarlas como actividades cotidianas de su desempeño. También, la empresa Timberland es un buen ejemplo de compañía con una sólida integridad de marca. Es bien conocida por el "Path of Service", un programa de servicio de voluntariado corporativo que involucra a empleados de la compañía (Kotler et al., 2010). Lo más interesante quizás, como señalan estos autores, no es el hecho en sí de que tengan este 
programa sino que la empresa entró en pérdidas en varios años consecutivos y muchos pensaban que esta programa desaparecería, pero los directivos consideraron que formaba parte del $\mathrm{ADN}$ de la empresa y el programa continúa en la actualidad (Austin et al., 2004).

En este sentido, uno de los objetivos de la RSC y el voluntariado corporativo es, de una parte, convencer a la sociedad de que las empresas pueden ayudar a combatir las desigualdades sociales que existen hoy en día y, de otra parte, conseguir la afinidad de más clientes por la realización de esta actividad y por la consideración hacia los más desfavorecidos.

Por tanto el voluntariado corporativo podría enmarcarse dentro de las estrategias de Creación de Valor Compartido (Porter y Kramer, 2011) que según estos autores suponen no solo compartir el valor ya creado por las empresas sino que se trata de aumentar de manera conjunta todo el caudal de valores económicos y sociales que puede generarse alrededor de las compañías. Las empresas, en muchos casos, mejor que los Gobiernos, pueden contribuir al desarrollo de la sociedad y su implicación en acciones de voluntariado corporativo con sus empleados $\mathrm{y} / \mathrm{o}$ clientes es una manera específica de concretar esa contribución.

Mientras que en Canadá más de los dos tercios de las empresas ya apoyan el voluntariado corporativo (Basilet et al., 2009), las primeras experiencias en España, las encontramos en la década pasada, promovidas fundamentalmente por las filiales de grupos internacionales. Especialmente bancos de inversión y empresas de servicios profesionales. En España, a principios del 2006, sólo ocho empresas del IBEX 35 promovían programas de voluntariado corporativo, a pesar de que, según datos de estudios de opinión realizados por la Fundación Empresa y Sociedad: al $85 \%$ de los ciudadanos que trabajan les gustaría participar en la acción social de su empresa; cerca del $50 \%$ de la ciudadanía prefiere empresas que dedican recursos a actividades de asistencia social (organizaciones que apoyan a personas con enfermedades terminales, a educación, a investigación, etc.); y más de la mitad de los trabajadores están a favor de que sus empresas colaboren con proyectos sociales (Sanz et al., 2010).

\subsection{Beneficios derivados del voluntariado corporativo}

Tal y como apunta Sanz et al.(2012), los beneficios para las empresas, empleados y para la sociedad derivados de las actividades de voluntariado corporativo son:

\section{Beneficios para los empleados}

El voluntariado corporativo parece incidir en la satisfacción, felicidad, compromiso y productividad del empleado, mejorando en general las actitudes del empleado hacia su trabajo (Peterson, 2004; FrankAlston, 2001). Varios autores han argumentado que las actividades de voluntariado se asocian positivamente con la satisfacción y el bienestar del individuo. De hecho, el trabajo voluntario está directamente vinculado a una mayor satisfacción con la vida y el bienestar, y una mayor participación del voluntariado (en términos de tiempo dedicado) también se relaciona con estos sentimientos (Farrell et al., 1998; Morrow-Howell et al., 2003; Wu et al., 2005; Do Paco et al., 2013).

Además, el voluntariado corporativo fomenta la solidaridad, lo que repercute no sólo dentro de la empresa, sino en los hogares y círculos sociales de los empleados y eleva la moral, generándoles un sentimiento de satisfacción por poder ayudar a otros (Sanz et al., 2012).

\section{Beneficios para las empresas}

Las empresas pueden beneficiarse directamente de su apoyo hacia el voluntariado corporativo de varias maneras. El voluntariado corporativo puede mejorar la imagen pública, las relaciones con los consumidores y las relaciones con la comunidad (Basil et al., 2011). Además, en múltiples estudios se ha analizado el impacto positivo que tienen los programas de voluntariado corporativo sobre los empleados, hasta el punto de que muchos, siempre que puedan elegir, prefieren trabajar en empresas que estén implicadas en la mejora de la comunidad (Caudron, 1994), por lo que también se le atribuye al voluntariado corporativo la captación de talento.

Por otro lado y en la misma forma que la persona individual se beneficia de las actividades de voluntariado, las empresas también obtienen ventajas de la situación, y puede incluso haber una correlación entre la satisfacción obtenida desde el trabajo voluntario y la facturación de las empresas (Watson y Abzug, 2005).

Además, también destacamos el sentimiento de orgullo que genera de pertenencia en los empleados y el hecho de que fortalece el vínculo entre la empresa y la comunidad en la que desarrolla su actividad (Sanz et al., 2012).

Beneficios para la Sociedad 
El voluntariado corporativo puede servir para que las empresas puedan tener una mejor relación con sus clientes y que éstos perciban la empresa desde esa otra óptica con lo que ayudaría a tener una mejor imagen de la marca. Indudablemente si los clientes entran en contacto con acciones de voluntariado gracias a la empresa, se les estaría ofreciendo una enriquecedora experiencia (Pine y Gilmore, 1988) y como estos mismos autores señalan, en muchos casos serían ocasión de directa para una transformación u oportunidad de mejora personal con el consiguiente beneficio para la sociedad.

Por otro lado, los beneficios que recibe la sociedad son una confluencia de todos los anteriores, pues la sociedad es, al fin y al cabo, la suma de todos aquellos factores y sucesos que se producen dentro de ella.

\subsection{El voluntariado corporativo en las empresas familiares}

A pesar de la crisis económica actual que afecta a muchas economías, las empresas familiares son hoy en día el modelo de negocio más extendido en cualquier estructura económica. De hecho, las empresas familiares en España representan el 85\% sobre el total de empresas y constituyen el $70 \%$ del PIB y del empleo privado (Instituto de la Empresa Familiar). Además, a pesar de que la investigación sobre empresa familiar avanza a buen ritmo (NúñezCacho, Grande y Muñoz, 2012) la Responsabilidad Social Corporativa constituye una dimensión de gran interés en el contexto de las empresas familiares que aún no ha sido suficientemente estudiado (Benavides et al., 2011).

Las empresas familiares también tratan de seguir estos modelos de negocio que consideran a la RSC como parte de su política y que están teniendo éxito en las grandes corporaciones. Además, mientras Graafland (2002) demostró que el nivel de preocupación de las empresas familiares por la RSC es mayor que el de las no familiares (Cabrera et al., 2011), otros autores encuentran que entre las empresas familiares, las que tienen estructura de consejos con más potencial para un comportamiento socialmente responsable (consejos profesionales) son las de mayor tamaño, edad, y evolución generacional (Cabrera et al., 2011).

Por tanto, es de interés para la comunidad científica y empresarial el análisis del voluntariado corporativo en la empresa familiar.

\subsection{Orientación al Mercado Interno y Dimensión Social de la RSC}

Como se ha visto, la implantación de un programa de voluntariado corporativo supondrá una nueva forma de gestión en las empresas, pero estos cambios pueden contribuir al éxito en la medida en que se gestione a partir de una filosofía de Orientación al Mercado Interno (Vázquez et al., 2010; Ruizalba et al, 2014; Gounaris, 2006). MohrJackson (1991) propone un modelo de Orientación al Mercado Interno en el que incluye tres actividades fundamentales: (1) entender las necesidades de los empleados (2) diseminar esa información entre departamentos e (3) incrementar los beneficios a los empleados para que se trasladen a los clientes externos. De igual modo, la dimensión social de la RSC, recoge aspectos tales como la mejora y el fomento de la calidad de vida de los empleados (Gallardo et al., 2013).

En primer lugar, la revisión de la literatura nos ha llevado a evidenciar la conveniencia de analizar cómo, las iniciativas actuales y posibles iniciativas futuras de la empresa sobre voluntariado corporativo, pueden afectar a dos actitudes clave: la satisfacción y el compromiso de los empleados.

Se persigue la satisfacción del empleado puesto que los clientes interactúan con los empleados (Kotler, 1997) y la satisfacción de los empleados se encuentra relacionada positivamente con la satisfacción de los clientes (Berry, 1987; Piercy, 1995). Por otro lado, Steers (1977) definió el compromiso como la fuerza de identificación y de involucración de un individuo con una organización. Las empresas valoran y persiguen elevar el nivel de compromiso de sus stakeholders, por ejemplo, sin el compromiso de los empleados es difícil llevar a la empresa a cumplir con su estrategia (Ruizalba, 2013).

Por lo tanto, planteamos las hipótesis de investigación siguientes:

H1a Las empresas que promuevan el voluntariado corporativo obtendrán mayores niveles de satisfacción por parte de sus trabajadores.

H1b Las empresas que promuevan el voluntariado corporativo obtendrán mayores niveles de compromiso por parte de sus trabajadores.

Además de la situación actual de implicación en el voluntariado corporativo, se considera oportuno valorar la intención de implantar dichas prácticas en un futuro, para de este modo, poder analizar su posible influencia sobre la satisfacción y el 
compromiso de los empleados. De este modo, se formulan las dos siguientes hipótesis:

H2a Las empresas que promuevan una futura implicación en voluntariado corporativo obtendrán mayores niveles de satisfacción por parte de sus trabajadores.

$\mathrm{H} 2 \mathrm{~b}$ Las empresas que promuevan una futura implicación en voluntariado corporativo obtendrán mayores niveles de compromiso por parte de sus trabajadores.

Por otro lado, y con la finalidad de poder comparar el grado de repercusión de dicha política de voluntariado corporativo, se añaden a dicho análisis otras variables concernientes a la dimensión social de la RSC muy relacionadas también con la Orientación al Mercado Interno. De este modo, estas otras políticas consideradas son: el interés que toma la dirección para satisfacer la necesidades generales de sus empleados y las políticas de conciliación entre la vida laboral y familiar implantadas.

En este sentido, uno de los factores analizados en relación a la Orientación al Mercado Interno, ha sido el management concern o interés de la dirección que se ha demostrado tener un impacto positivo sobre el compromiso y la satisfacción de los empleados (Ruizalba et al., 2014). Eisenberger et al. (1986) desarrollaron una interesante investigación y apuntan que si las personas perciben que la organización se interesa por ellas, procura su bienestar y les brinda ayuda ante problemas personales cuando es necesario, las consecuencias son muy favorables para la organización en lo que se refiere a mejor desempeño y deseos de permanencia. En consecuencia, se proponen como hipótesis:

H3a: Las empresas que potencien el interés que se toma la dirección en satisfacer las necesidades del empleado obtendrán mayores niveles de satisfacción por parte de estos

H3b Las empresas que potencien el interés que se toma la dirección en satisfacer las necesidades del empleado obtendrán mayores niveles de compromiso por parte de estos

Por último, la conciliación entre la vida laboral, personal y familiar se está analizando con más frecuencia en relación a la satisfacción laboral y al compromiso de los empleados, tanto desde el ámbito del marketing interno (Ruizalba et al, 2014; Ruizalba y Vallespín, 2014) como desde el ámbito de dirección de empresas (Thomas y Ganster, 1995; Thomson y Prottas, 2005). En este sentido, el informe IFREI (IFREI, 2011) ha concluido que el compromiso de los empleados es cuatro veces más elevado en aquellas compañías en las que el ámbito de trabajo es favorecedor hacia la práctica de la conciliación, mientras que por el contrario, la insatisfacción laboral aumenta once veces más en aquellas empresas que dificultan la práctica de la conciliación. En base a lo cual podemos proponerlas siguientes hipótesis:

H4a Las empresas que potencien la conciliación de la vida laboral con la familiar obtendrán mayores niveles de satisfacción por parte de sus trabajadores.

H4b Las empresas que potencien la conciliación de la vida laboral con la familiar obtendrán mayores niveles de compromiso por parte de sus trabajadores.

\section{Metodología}

La técnica cuantitativa de apoyo para el desarrollo de esta investigación ha sido la encuesta. Esta encuesta se dirigió a informantes claves de empresas familiares andaluzas. La decisión de elegir al informante clave proviene del hecho de que se trata de un directivo que posee la visión global de la organización y la capacidad necesaria para responder con conocimiento de causa a las preguntas planteadas en el cuestionario. Para la recogida de información, se utilizó el envío de email con enlace al cuestionario online con apoyo en algunos casos de llamadas telefónicas.

En la tabla 1 se presenta la ficha técnica del trabajo de campo llevado a cabo.

\section{Tabla 1}

Ficha técnica

\begin{tabular}{ll}
\hline Ámbito geográfico & Andalucía \\
$\begin{array}{l}\text { Población } \\
\text { Tipo de muestreo }\end{array}$ & $\begin{array}{l}\text { Muestreo de conveniencia } \\
\text { Tamaño de la } \\
\text { muestra }\end{array}$ \\
$\begin{array}{l}\text { Modo de aplicación empresas familiares } \\
\text { Correo electrónico con encuesta } \\
\text { online y apoyo telefónico }\end{array}$ \\
$\begin{array}{l}\text { Fecha de } \\
\text { realización }\end{array}$ & \begin{tabular}{l} 
Marzo- Septiembre, 2013 \\
\hline
\end{tabular}
\end{tabular}

Por otro lado, aunque existe cierto consenso en relación con el concepto de empresa familiar; al tratarse de un concepto multifactorial y multidisciplinar, no está exento de discusión su tratamiento (Rojo et al., 2011; Benavides et al., 2011). De este modo, para una correcta 
caracterización de las empresas familiares se formularon dos preguntas que la literatura considera correctas para su conceptualización (Comblé y Colot, 2006; Rojo et al., 2011): el control del capital por la familia y la participación activa de la familia en la gestión de la empresa. Por lo tanto, considerando empresa familiar a aquellas que cumplan ambos requisitos, estamos también ajustándonos a dos de los criterios básicos, propiedad accionarial y gobierno de la empresa, que establece el Instituto Familiar para considerarse empresa familiar.

Para conocer la población total de empresas familiares en Andalucía se parte de la base de datos del Dirce (2012) y del dato estimado por el Instituto de Empresa Familiar de que el $85 \%$ de las empresas españolas son familiares. A partir de este dato y mantenido la misma proporción de empresas familiares en cada una de las provincias respecto al total de empresas (según datos del Dirce) nos quedaría la distribución de empresas familiares por provincias andaluzas que se presentan en la tabla 2. Dicha proporción y a pesar de tratarse de un muestreo no probabilístico, es la que se ha perseguido para que quedara reflejada en nuestra muestra.

Tabla 2

Número de empresas encuestadas por provincia andaluza

\begin{tabular}{lll}
\hline Provincias & $\begin{array}{l}\text { Número de } \\
\text { empresas }\end{array}$ & $\begin{array}{l}\text { Porcentaje de empresas por } \\
\text { provincia sobre el total de } \\
\text { Andalucía }\end{array}$ \\
\hline Almería & 6 & $9 \%$ \\
Cádiz & 8 & $13 \%$ \\
Córdoba & 6 & $10 \%$ \\
Granada & 7 & $11 \%$ \\
Huelva & 3 & $5 \%$ \\
Jaén & 5 & $7 \%$ \\
Málaga & 14 & $22 \%$ \\
Sevilla & 15 & $23 \%$ \\
Total & 64 & $100 \%$ \\
\hline
\end{tabular}

El cuestionario se estructuró en 2 partes:

El primer bloque recoge 16 preguntas relacionadas con aspectos de la dimensión social de la RSC. Las variables se plantean como afirmaciones en escalas Likert de 7 puntos, desde 1 (totalmente en desacuerdo) hasta 7 (totalmente de acuerdo) sobre las que se pide opinión al informante clave.
En el segundo bloque se incluyen cuestiones relativas a las empresas para su clasificación: 2 preguntas de confirmación acerca de su naturaleza de empresa familiar, el nombre de la empresa, la generación en la que se encuentra, el sector y la provincia andaluza en la que opera y el número de empleados.

Para la elaboración de dicho cuestionario se han tomado como referencia trabajos anteriores resultantes de la revisión teórica realizada. Fundamentalmente se toma como referencia los trabajos de Ruizalba et al. (2014); Gounaris (2008); Clark (2001); Hartline y Ferrel (1996); Kossek y Nichol (1992) y Meyer y Allen (1991).

Por último y para asegurarnos de la idoneidad de nuestro cuestionario, se realizó un pretest en el que se seleccionaron a 5 empresarios propietarios de empresas familiares con más de 10 años de experiencia y a 5 académicos especialistas en investigaciones del ámbito que nos ocupa.

\section{Análisis y discusión de resultados}

\subsection{Dimensionalidad y fiabilidad de las escalas}

El primer paso ha consistido en un análisis de naturaleza exploratoria para el estudio de la fiabilidad y dimensionalidad de las escalas, mediante dos análisis factoriales de componentes principales y rotación Varimax.

En la tabla 3, se recogen los resultados obtenidos para la escala concerniente a la dimensión social de la RSC. El valor 0,71 de la medida de adecuación muestral KMO, junto a los coeficientes alfa de Cronbach, indican que los resultados que a continuación se analizan son adecuados y que, por tanto, se podrán resumir las 10 variables en cuatro dimensiones. De este modo, los ítems se agrupan en cuatro dimensiones que explican el $85 \%$ de la varianza total. El primer concepto (ID) está formado por 3 items relacionados con el interés que muestra la dirección por satisfacer las necesidades de sus empleados como las políticas llevadas a cabo, el tiempo o el dinero invertido. El segundo (CVLF) agrupa 3 items referidos al grado de facilidades que ofrece la empresa para que sus trabajadores concilien su vida profesional con su vida familiar. El tercer concepto (IAVC) hace referencia a la implicación actual de la empresa respecto al voluntariado corporativo, tanto para sus trabajadores como para sus clientes. Para finalizar, el cuarto factor (IFVC) recoge 2 items relacionados con la 
Tabla 3

Análisis de fiabilidad y dimensionalidad.

\begin{tabular}{|c|c|c|c|}
\hline \multicolumn{2}{|c|}{ Dimensión social de la RSC } & $\begin{array}{l}\text { Cargas } \\
\text { Factoriales }\end{array}$ & $\begin{array}{l}\text { Varianza } \\
\text { explicada y } \\
\text { alfa de } \\
\text { Cronbach }\end{array}$ \\
\hline \multirow{3}{*}{$\begin{array}{l}\text { ID: Interés de } \\
\text { la dirección }\end{array}$} & $\begin{array}{l}\text { Los directivos de esta empresa invierten recursos (tiempo y/o } \\
\text { dinero) si hay algo que se pueda hacer para satisfacer las } \\
\text { necesidades específicas o los requerimientos de los empleados }\end{array}$ & 0,91 & \multirow{3}{*}{$\begin{array}{l}21,06 \% \\
\alpha=0,87\end{array}$} \\
\hline & $\begin{array}{l}\text { Las necesidades de los empleados se toman en serio por la } \\
\text { dirección de la empresa y se introducen políticas para satisfacerlas. }\end{array}$ & 0,74 & \\
\hline & $\begin{array}{l}\text { La dirección de esta empresa está claramente orientada a la } \\
\text { resolución de los problemas de los empleados y a dotarles de todo } \\
\text { el apoyo necesario para que puedan desempeñar bien su trabajo }\end{array}$ & 0,68 & \\
\hline \multirow{3}{*}{$\begin{array}{l}\text { CVLF: } \\
\text { Conciliación } \\
\text { Vida Laboral } \\
\text { y Familiar }\end{array}$} & $\begin{array}{l}\text { Los directivos comprenden las necesidades familiares de los } \\
\text { empleados. }\end{array}$ & 0,70 & \multirow{3}{*}{$\begin{array}{l}25,56 \% \\
\alpha=0,86\end{array}$} \\
\hline & $\begin{array}{l}\text { Los directivos apoyan a los empleados para que puedan compaginar } \\
\text { el trabajo y con sus responsabilidades familiares }\end{array}$ & 0,91 & \\
\hline & $\begin{array}{l}\text { En esta empresa los trabajadores pueden tener un equilibrio entre el } \\
\text { trabajo y sus vidas familiares. }\end{array}$ & 0,83 & \\
\hline \multirow{2}{*}{$\begin{array}{l}\text { IAVC: } \\
\text { Implicación } \\
\text { actual en } \\
\text { Voluntariado } \\
\text { Corporativo }\end{array}$} & $\begin{array}{l}\text { Esta empresa promueve que sus empleados se impliquen en } \\
\text { actividades de voluntariado }\end{array}$ & 0,91 & \multirow{2}{*}{$\begin{array}{l}19,07 \% \\
\alpha=0,89\end{array}$} \\
\hline & $\begin{array}{l}\text { Esta empresa promueve que sus clientes se impliquen en } \\
\text { actividades de voluntariado }\end{array}$ & 0,95 & \\
\hline \multirow{2}{*}{$\begin{array}{l}\text { IFVC: } \\
\text { Implicación } \\
\text { futura en } \\
\text { Voluntariado } \\
\text { Corporativo }\end{array}$} & $\begin{array}{l}\text { Nos gustaría implicarnos más para que nuestros empleados } \\
\text { realizaran más actividades de voluntariado }\end{array}$ & 0,95 & \multirow{2}{*}{$\begin{array}{l}19,67 \% \\
\alpha=0,95\end{array}$} \\
\hline & $\begin{array}{l}\text { Nos gustaría implicarnos más para que nuestros clientes realizaran } \\
\text { más actividades de voluntariado }\end{array}$ & 0,94 & \\
\hline
\end{tabular}

$\mathrm{KMO}=0,719$ Prueba de esfericidad de Barlett $\mathrm{X}^{2}(45)=409,38(\mathrm{p}=0,000)$ Residuos $=15 \%$

intención futura de la empresa para promover el voluntariado corporativo.

Por otro lado, en la tabla 4 , se recogen los resultados obtenidos para la escala concerniente a los niveles de satisfacción y compromiso de los empleados. El valor 0,78 de la medida de adecuación muestral KMO, junto a los coeficientes Alfa de Cronbach, indican la dimensionalidad del constructo actitudes. Los ítems se agrupan en dos dimensiones, Satisfacción y Compromiso que explican el $86,68 \%$ de la varianza total.

\subsection{Caracterización de la muestra}

La muestra obtenida se ha conformado por 64 empresas familiares andaluzas de todo el territorio de la Comunidad Autónoma, de las 8 provincias. En nuestra muestra de empresas familiares andaluzas, un $26,6 \%$ son empresas familiares de primera generación, un $54,7 \%$ son de segunda generación y el $6,3 \%$ son ya de $3^{\circ}$ generación.

Por otro lado, el 12,5\% tienen entre 1 y 9 empleados, el $18,8 \%$ entre 30 y 49 , el $15,6 \%$ entre 50 y 199 y tan solo el 3,1\% afirma tener más de 200 empleados.

Además se desprende que aproximadamente el $60 \%$ de ellas se dedican al sector servicios, como empresas del ámbito del turismo, de la formación y comerciales fundamentalmente. Representativas de otros sectores, conforman un 7,8\% del ámbito de la construcción y un $5 \%$ de empresas productoras y de transformación.

\subsection{Indicadores básicos}

En la tabla 5, se muestran los estadísticos descriptivos que responden a algunos indicadores sobre el ámbito de la dimensión social de la RSC. Se 
Tabla 4

Análisis de fiabilidad y dimensionalidad

\begin{tabular}{|c|c|c|c|}
\hline Actitudes & & $\begin{array}{l}\text { Cargas } \\
\text { Factoriales }\end{array}$ & $\begin{array}{l}\text { Varianza explicada y alfa } \\
\text { de Cronbach }\end{array}$ \\
\hline \multirow{3}{*}{ SAT: Satisfacción } & $\begin{array}{l}\text { Estoy satisfecho de las relaciones con mis } \\
\text { jefes }\end{array}$ & 0,86 & \multirow{3}{*}{$\begin{array}{l}55,09 \% \\
\alpha=0,87\end{array}$} \\
\hline & $\begin{array}{l}\text { Estoy satisfecho del apoyo que recibo de la } \\
\text { empresa }\end{array}$ & 0,89 & \\
\hline & $\begin{array}{l}\text { Estoy satisfecho de las oportunidades de } \\
\text { avanzar en esta empresa }\end{array}$ & 0,83 & \\
\hline \multirow{2}{*}{ COM: Compromiso } & $\begin{array}{l}\text { Me percibo emocionalmente ligado a la } \\
\text { empresa }\end{array}$ & 0,66 & $31,59 \%$ \\
\hline & $\begin{array}{l}\text { Me sentiría algo culpable si tuviera que dejar } \\
\text { ahora la empresa }\end{array}$ & 0,95 & $\alpha=0,85$ \\
\hline
\end{tabular}

$\mathrm{KMO}=0,783$ Prueba de esfericidad de Barlett $\mathrm{X}^{2}(10)=231,73(\mathrm{p}=0,000)$ Residuos $=30 \%$

observa como las políticas de conciliación entre la vida laboral y familiar están siendo bastante llevadas a cabo por las empresas familiares andaluzas, con una media de 5,16. Respecto al interés que muestra la dirección por satisfacer las necesidades de los empleados existen menos diferencias entre unas y otras empresas que con respecto a otras políticas sociales.

Por otro lado, resulta aún escaso el número de empresas que están llevando políticas de implicación en voluntariado corporativo $(2,96)$ aunque parece que es un tema que empiezan a plantearse de cara al futuro $(5,1)$. Por lo tanto, parece que las empresas perciben con nitidez la importancia que pueden llegar a tener los aspectos sociales en el proceso de creación de valor, y en consecuencia, piensan incorporar el voluntariado corporativo en los próximos años. También se observa que el voluntariado con empleados es el que se está llevando más a cabo $(3,06)$ pero que en un futuro, las empresas también están considerando su orientación hacia el cliente final.

\subsection{Estimación de un modelo de regresión}

Además, se ha hecho uso del análisis de regresión lineal múltiple que permite examinar la relación entre las distintas variables, determinando la influencia de las variables independientes sobre la dependiente (McDaniel y Gates, 1999). En nuestro caso, se han desarrollado dos modelos de regresión tomando en la primera, como variable dependiente, la satisfacción del empleado, y en la segunda, su compromiso hacia la empresa. Sobre estas se regresan el conjunto de variables explicativas (interés de la dirección, conciliación de la vida laboral y familiar, implicación actual en voluntariado corporativo e implicación futura en voluntariado corporativo).

Para corregir el problema de multicolinealidad con el que nos encontramos (se observan multitud de correlaciones estadísticamente significativas), acudimos a utilizar los factores basados en la estimación del análisis factorial como variables de entrada en la regresión (Hair et al., 2007).

De este modo, y a pesar de la pérdida de información que supone, se resumen las variables en una serie de factores ortogonales con menor correlación entre ellos, solventando el incumplimiento del supuesto básico y obteniendo una ausencia total de multicolinealidad (valores próximos a 1 en el índice de tolerancia y del Factor de Inflación de la varianza). En el caso de la regresión múltiple, y teniendo en cuenta que utilizamos datos de corte transversal, debe haber tanto linealidad entre las variables explicativas y la explicada (se comprueba gráficamente la hipótesis de linealidad).

De igual modo se verifica, a través del gráfico Q$\mathrm{Q}$ normal la hipótesis de normalidad y con D-W la hipótesis de ausencia de autocorrelación. 
Tabla 5

Análisis descriptivo.

\begin{tabular}{lcll}
\hline Variables (indicadores globales) & Media & Desviación típica & Rango \\
\hline Interés de la dirección & 4,95 & 1,11 & 5,33 \\
Conciliación Vida Laboral y Familiar & 5,16 & 1,12 & 6 \\
Implicación actual en Voluntariado Corporativo & 2,96 & 1,74 & 6 \\
Con empleados & 3,06 & & \\
Con clientes & 2,81 & & 6 \\
Implicación futura en Voluntariado Corporativo & 5,1 & 1,90 & \\
Con empleados & 5,08 & & \\
Con clientes & 5,25 & & \\
\hline
\end{tabular}

La tabla 6 muestra los resultados de la regresión realizada para explicar la satisfacción de los empleados. Se observa como el estadístico $\mathrm{F}(\mathrm{F}=$ $11,260)$ es significativo para un nivel de $\mathrm{p}<0,000$. Ahondando en el detalle de la regresión, cabe destacar el nivel que el R2 ajustado muestra $(0,45)$. Los datos indican que 3 variables son significativas, siendo la variable más importante a la hora de explicar la satisfacción de los empleados el Interés de la Dirección.

La variable que no resulta significativa para la satisfacción del personal es su implicación futura en los programas de Voluntariado Corporativo. Por tanto, no podemos aceptar la hipótesis 2a (aceptando 1a, 3a y 4a). Efectivamente, parece que los planes de futuro no afectan a la satisfacción del empleado aunque sí todo lo concerniente a lo que la empresa está realizando en la actualidad respecto a la dimensión social de su RSC.
De manera análoga, la tabla 7 muestra los resultados de la regresión realizada para explicar el compromiso de los empleados. De igual modo, se observa como el estadístico $\mathrm{F}$ es significativo para un nivel de $\mathrm{p}<0,0001$ y mostrando un R2 ajustado de 0,40 . Los datos indican que solo dos de las cuatro variables son significativas.

En primer lugar, parece que la variable más influyente vuelve a ser el interés que muestra la Dirección por satisfacer algunas de las necesidades de sus empleados y por otro, el grado de implicación actual en voluntariado corporativo.

Es interesante destacar el hecho de que sea la variable de voluntariado corporativo y no la referente a la conciliación de la vida laboral y familiar. Por lo tanto, no se pueden aceptar las hipótesis $2 \mathrm{~b}$ y $4 \mathrm{~b}$.

\section{Tabla 6}

Análisis de regresión sobre la satisfacción de los empleados

\begin{tabular}{|c|c|c|c|c|c|}
\hline Variable & Media & Desv. & $\begin{array}{l}\text { Coef. } \\
\text { Regres. TIP. }\end{array}$ & Error Estandard & T-Student \\
\hline ID: Interés de la dirección & 0,000 & 1 & 0,494 & 0,145 & $\begin{array}{l}4,940 \\
(p=0,000)\end{array}$ \\
\hline $\begin{array}{l}\text { CVLF: Conciliación Vida } \\
\text { Laboral y Familiar }\end{array}$ & 0,000 & 1 & 0,257 & 0,145 & $\begin{array}{l}2,567 \\
(p=0,013)\end{array}$ \\
\hline $\begin{array}{l}\text { IAVC: Implicación actual en } \\
\text { Voluntariado Corporativo }\end{array}$ & 0,000 & 1 & 0,367 & 0,145 & $\begin{array}{l}3,67 \\
(p=0,001)\end{array}$ \\
\hline $\begin{array}{l}\text { IFVC: Implicación futura en } \\
\text { Voluntariado Corporativo }\end{array}$ & 0,000 & 1 & $-0,073$ & 0,145 & $\begin{array}{l}-0,731 \\
(\mathrm{p}=0,468)\end{array}$ \\
\hline
\end{tabular}


Tabla 7

Análisis de regresión sobre el compromiso de los empleados.

\begin{tabular}{|c|c|c|c|c|c|}
\hline Variable & Media & Desv. & $\begin{array}{l}\text { Coef. Regres. } \\
\text { TIP. }\end{array}$ & Error Estandard & T-Student \\
\hline ID: Interés de la dirección & 0,000 & 1 & 0,460 & 0,144 & $\begin{array}{l}4,425 \\
(p=0,000)\end{array}$ \\
\hline $\begin{array}{l}\text { CVLF: Conciliación } \quad \text { Vida } \\
\text { Laboral y Familiar }\end{array}$ & 0,000 & 1 & 0,143 & 0,144 & $\begin{array}{l}1,376 \\
(p=0,174)\end{array}$ \\
\hline $\begin{array}{l}\text { IAVC: Implicación actual en } \\
\text { Voluntariado Corporativo }\end{array}$ & 0,000 & 1 & 0,416 & 0,144 & $\begin{array}{l}4,003 \\
(p=0,000)\end{array}$ \\
\hline $\begin{array}{l}\text { IFVC: Implicación futura en } \\
\text { Voluntariado Corporativo }\end{array}$ & 0,000 & 1 & $-0,008$ & 0,144 & $\begin{array}{l}-0,076 \\
(p=0,939)\end{array}$ \\
\hline
\end{tabular}

D-W: 1,78

\section{Conclusiones, limitaciones y propuestas}

La investigación realizada permite analizar el impacto que algunas prácticas relacionadas con la dimensión social de la RSC y la Orientación al Mercado Interno tienen sobre los empleados, en concreto, sobre su satisfacción laboral y sobre su compromiso organizacional.

Una de las conclusiones extraídas es que en aquellas empresas con mayor presencia del voluntariado corporativo, la satisfacción laboral y el compromiso de los empleados es mayor. Por tanto, el hecho de que las empresas pongan en marcha acciones de voluntariado corporativo podría contribuir a incrementar los niveles de satisfacción laboral y del compromiso de los empleados. Es decir, las empresas podrían conseguir una ventaja competitiva a través de estas acciones, sin la necesidad de invertir grandes presupuestos. Además, como se ha visto, el interés de la dirección y las acciones de voluntariado, tienen un peso importante a la hora de explicar el nivel de satisfacción laboral y el compromiso, así que el desarrollo de ambos factores conjuntamente podrían contribuir a potenciar la satisfacción laboral y el compromiso.

También como propuestas para la práctica empresarial, las empresas podrían diseñar no solo acciones de voluntariado corporativo pensando en los empleados sino también diseñarlas con el objetivo de intentar involucrar a sus clientes, pues de los resultados empíricos se observa que evolucionan paralelamente las intenciones de implantar el voluntariado en ambos grupos de interés. No obstante, hay que matizar que no en todos los sectores ni empresas sería posible o deseable involucrar a los clientes en estos planes por lo que un análisis de la situación sería recomendable caso a caso.

Otra de las propuestas para la práctica empresarial es que el voluntariado corporativo sea tenido en cuenta en el marco de las prácticas de marketing interno, en concreto en la subdimensión que la literatura anglosajona denomina "management concern" y que se podría traducir como Interés de la Dirección y que tiene un importante papel en el desarrollo del compromiso organizativo (Ruizalba, 2013).

Teniendo en cuenta que el voluntariado que llevan a cabo se relaciona con una mayor retención, satisfacción laboral, y construcción de equipo (Boccalandro, 2009) esto refuerza la conveniencia de su implantación por parte de las empresas. Además el voluntariado corporativo también ha demostrado ayudar a desarrollar destrezas y competencias, como la comunicación, el trabajo en equipo y el liderazgo (City of London 2010; Tuffrey 1998).

Una limitación de esta investigación parte del hecho de haberla realizado solo en el ámbito de la Comunidad Autónoma de Andalucía (España). Otra limitación podría provenir del hecho de solo haber analizado algunas de las variables que inciden en la dimensión social de la RSC. Por último, también encontramos como posible limitación el que no se haya profundizado en el tipo de voluntariado sobre el que se está encuestando, en este sentido, algunos autores como Peterson (2004) han distinguido entre voluntariado prestado en programas organizados o coordinados por la propia compañía o bien por entidades ajenas a la empresa en la que se trabaja.

De este modo, como propuestas para futuras líneas de investigación, se señalan extender el 
estudio en relación a la clase de voluntariado implantado en las empresas y de este modo estudiar si se trata de programas organizados por la propia empresa o bien por entidades ajenas; también resultaría interesante poder clasificar el tipo de voluntariado en función de su naturaleza y beneficiarios (medioambiental, asistencial, educativo, etc). Además sugerimos que se pueda extender un análisis similar a otras áreas de la geografía española así como la realización de un análisis comparativo entre empresas familiares y otro tipo de empresas.

Por último y como propuesta para el ámbito académico advertir de la necesidad de desarrollar más investigaciones en el ámbito de la empresa familiar y desde el campo de la RSC y de la ética. Según Benavides et al. (2011) desde el año 1961 a 2008, sobre los 684 artículos que analizaron incluidos en las categorías «business», «business finance» y «management» en la edición de 2008 del Journal Citation Report (JCR) del Social Science Citation Index (SSCI), solo ha habido 8 artículos con esta temática. Estos mismos autores señalan que en el mismo período tan solo ha habido 4 artículos sobre Marketing en empresas familiares, lo cual nos incita a proponer la realización de más trabajos de investigación sobre Marketing, y en concreto, proponemos sobre Marketing Interno pues en esta investigación hemos visto la relación del voluntariado corporativo con algunas políticas de Marketing Interno.

\section{Bibliografía}

Austin, J., Leonard, H. y Quinn, J. (2004). Timberland: Commerce and Justice. Harvard Business School Case.

Asociación Española de Contabilidad y Administración de Empresas (2003). Marco conceptual de la responsabilidad social corporativa. Documentos AECA. Serie Responsabilidad Social Corporativa. Documento numero 1, Madrid.

Basil, D. Z., Runte, M. S., Easwaramoorthy, M y Barr, T. (2008). Company Support for Employee Volunteering: A National Survey of Companies in Canada, Journal of Business Ethics, 85, 387-398.

Basil, D., Runte, M., Basil, M. y Usher, J. (2009). Company support for employee volunteerism: Does size matter? Journal of Business Research, 64, 61-66

Benavides, C.; Guzmán, V. y Quintana, C. (2011). Evolución de la literatura sobre empresa familiar como disciplina científica. Cuadernos de Economía y Dirección de la Empresa, 14, 78-90.
Berry, L. (1987). Big ideas in services marketing, Journal of Services Marketing, 1 (1), 5 - 9

Boccalandro, B. (2009). Mapping success in employee volunteering: The drivers of effectiveness for employee volunteering and giving programs and fortune 500 performance, Boston College Center for Corporate Citizenship,

Cabrera, M.K.; Déniz, M.C. y Martín, J.D (2011). Consejos de administración y potencial para la responsabilidad social de las empresas familiares no cotizadas españolas. Investigaciones Europeas de Dirección y Economía de la Empresa, 17 (3), 47-67.

Caligiuri, P.; Mencin, A. y Jiang, K. (2013). Win-winwin: The influence of company- sponsored volunteerism programs on employees, NGOs, and business units. Personnel Psychology, 66, 825-860.

Caudron, S. (1994). Volunteer efforts offer low-cost training options, Personnel Journal, Vol.73, No.6, pp.3844.

Carroll, A. B. (1991). The pyramid of corporate social responsibility: toward the moral management of organizational stakeholders. Business Horizons, 34(4), $39-48$.

Carroll, A. B. (1999). Corporate social responsibility. Business and Society, 38(3),268-295.

Chrisman, J.J., Chua, J.H. y Sharma, P. (2005). Trends and directions in the development of a strategic management theory of the family firm. Entrepreneurship Theory and Practice 29, 555-575.

Comblé, K. y Colot, O. (2006). L'entreprise familiale: concept et importance en belgique. reflets et perspectives. XLV(2), 91-108.

Dirce (2012): Directorio Central de Empresas. INE (Instituto Nacional de Estadística de España).

Clark, S.C. (2001). Work cultures and work/family balance, Journal of Vocational Behaviour, 58, 348-365

City of London (2010). Volunteering-The business case.

Do Paco, A. y Nave, A. C. (2013). Corporate volunteering A case study centred on the motivations, satisfaction and happiness of company employees. Employee relations, 35, 5, 547-559.

Eisenberger, R., Hungtington, R; Hutchinson, S. y Sowa, D. (1986). Perceived Organizational Support. Journal of Applied Pshychology, 71, 500-507.

Frank-Alston, M. (2001). The influence of community service/volunteer work on perceptions of job satisfaction, job motivation, and organizational commitment on employees in a manufacturing plant, unpublished doctoral disertation.

Freeman, R. E. (1984). Strategic management: A stakeholder approach. Boston, Massachusetts: Pitman Publishing. 
Gallardo, D.; Sánchez, M.I.; Corchuelo, M.B. y Guerra, A. (2010). Diagnóstico del voluntariado corporativo en la empresa española. Revista de Estudios Empresariales. Segunda época, 2, 54-80.

Gallardo-Vázquez, D., Sánchez-Hernández, M.I. y Corchuelo-Martinez-Azua, M.B (2013). Validación de un instrumento de medida para la relación entre la orientación a la responsabilidad social corporativa y otras variables estratégicas de la empresa. Revista de Contabilidad - Spanish Accounting Review, 16 (1), 11-23.

Gómez, Lumbreras, Martí y Rodríguez-Colubi, (2011). Voluntariado Corporativo en España. Informe 2011. Observatorio de Voluntariado Corporativo.

Gounaris, S. (2006). Internal-market orientation and its measurement. Journal ofBusiness Research 59, 432448 .

Gounaris, S. (2008). The notion of internal market orientation and employee job satisfaction: some preliminary evidence", Journal of Services Marketing, 22 (1), 68-90.

Graafland, J.J. (2002). Corporate Social Responsibility and Family Business, Paper presented at the Research Forum of the Family Business Network 13th Annual Conference, Helsinki, Finland.

Greenfield, E.A. y Marks, N.F. (2004). Formal volunteering as a protective factor of older adult's psychological well-being". Journal of Gerontology, 59B (5), 5258-5264.

Hair, J.F.; Anderson, R.E.; Tatham, R.L. y Black, W.C. (2007). Análisis multivariante. 5a: Pearson- Prentice Hall.

Hartline, M. y Ferrel, O.C. (1996). The management of customercontact service employees: an empirical investigation. Journal of Marketing, 60 (4), 52-70.

Hawkins, D., y Mann S. (2007). The World Bank's Role in Tourism Development. Annals of Tourism.Research, 34, 348-363.

IFREI (2011). Estudio sobre los efectos de la conciliación en el compromiso y la productividad. International Center for Work and Family, IESE Business School.

Joyner, B. E., y Payne, D. (2002). Evolution and Implementation: A study of values, business ethics and corporate social responsability. Journal of Business Ethics, 41(4), 297-311. Kotler, P. (1997): Marketing Management, Prentice-Hall, Englewood Cliffs, NJ.

Kossek, E.E. y Nichol, V. (1992). The effects of onskite child care on employee attitudes and performance. Personnel Psychology, 45, 485-509.

Kotler, P. (1997). Marketing Management, PrenticeHall, Englewood Cliffs, NJ.
Kotler, P., Kartajaya, H. y Setieawan, I. (2010). Marketing 3.0: from products to consumers to human spirit. Ed.John Wiley \& Sons.

McDaniel, C. y Gates, R. (1999). Investigación de Mercados Contemporánea, Thomson Editores.

Meyer, J.P. y Allen y Natalie, J. (1991). A three component conceptualization of organizational commitment. Human Resource Management Review, 1, 61-98.

Mohr-Jackson, I. (1991). Broadening the market orientation: an added focus on internal customers. Human Resource Management, 30(4), 455-467

Moon, J. (2011). Corporate social responsibility: an idea whose time has come? If so, what idea? Management and Business: Fifty Years On (a conference in recognition of Grigor McClelland). Manchester BusinessSchool.

Morrow-Howell, N., Hinterlong, J., Rozario, P.A. y Tang, F. (2003). Effects of volunteering on the well-being of older adults. Journal of Gerontology, 58B (3), S137S145.

Nieto, M. y Fernández, R. (2004). Responsabilidad social corporativa: la última innovación en management. Universia Business Review, 1, 28-39.

Núñez-Cacho, P.; Grande, F. y Muñoz, A. (2012). Rendimiento en las empresas familiares desde las teorías de recursos y capacidades y de agencia. Revista de empresa familiar, 2(1), 7-20.

Peterson, D. (2004). Benefits of participation in corporate volunteer programs: employees perceptions. Personnel Review, 33(5), 615- 627.

Piercy, N. (1995). Customer Satisfaction and the Internal Market: Marketing our Customers to our Employees, Journal of Marketing Practice and Applied Marketing Science, 1, 22-24.

Pine, J.; Gilmore, J (1998). "Welcome to the Experiencie Economy”. Harvard Business Review. JulyAugust.

Porter, M.; Kramer, M. (2011). Creating Shared Value: How to Reinvent Capitalism and Unleash Wave of Innovation and Growth. Harvard Business Review, 89(12), 62-77.

Reynolds, P. (2013). Hotel companies and corporate environmentalism. Tourism \& Management Studies, 9 (1), 7-12.

Rojo, A.; Diéguez, J. y López, P. (2011). Importancia del concepto de empresa familiar en investigación: utilización de la base de datos SABI para su clasificación. Revista de Empresa Familiar, 1 (1), 53-67.

Ruizalba J. (2013). El compromiso organizacional: un valor personal y empresarial en el marketing interno. Revista de Estudios Empresariales. Segunda época, 1, 6786. 
Ruizalba, J.L; Bermúdez, G.; Rodríguez, M. y Blanca, M.J. (2014). Internal Market Orientation: an empirical research in hotel sector. International Journal of Hospitality Management, 38, 11-19.

Ruizalba, J.L. y Vallespín, M. (2014). Empirical analysis of the constituent factors of internal marketing orientation at Spanish hotels. Tourism \& Management Studies, 10, 151-157.

Sanz, B., Cordobés M. y Calvet A. (2012). El voluntariado corporativo en España. Modelos y perspectivas de impacto social. Instituto de Innovación Social, ESADE.

Servero, R. y Villalonga I. (2005). La responsabilidad social corporativa y su gestión integrada. Centro de Investigacion y Especializacion en gestión de empresas Agroalimentarias. Universidad Politécnica de Valencia.

Steers, R.M. (1977). Antecedents and outcomes of organizational commitment. Administrative Science Quarterly, 22, 46-56.

Thomas, L.T. y Ganster, D.C. (1995). Impact of family-supportive work variables onwork-family conflict and strain: a control perspective. Journal of Applied Psychology, 80 (1), 6-15.

Thompson, C.A. y Prottas, D. (2005). Relationships among organizational family sup-port, job autonomy, perceived control, and employee well-being. Journal of Occupational Health Psychology 11 (1), 100-118

Tuffrey, M. (1998). Valuing employee community involvement: Practical guidance on measuring the business benefits from employee involvement in community activity," The Corporate Citizenship Company.

Watson, M.R. y Abzug, R. (2005). Finding the ones you want, keeping the ones you find",en Herman, R.D. et al. (Eds), Handbook of Nonprofit Leadership \& Management, Jossey-Bass, San Francisco, CA, 623-660.

Wild, C. (1993). Corporate Volunteer Programs: Benefits to Business, Report No.1029. The Conference Board, New York.

Wu, A.M.S., Tang, C.S.K. y Yan, E.C.W. (2005). Post-retirement voluntary work and psychological functioning among older Chinese in Hong Kong. Journal of Cross-Cultural Gerontology, 20 (1), 27-45. 